\title{
Detection of colistin-resistant Gram-negative rods by using the SuperPolymyxin medium
}

\author{
Aurélie Jayol a,b,c,d, Laurent Poirel ${ }^{\text {c,d,e, },, 1}$, Catherine André b ${ }^{\text {, }}$ Véronique Dubois ${ }^{\text {a,b }}$, Patrice Nordmann ${ }^{\text {c,d,e,f }}$ \\ a Laboratory of Bacteriology, Bordeaux University Hospital, Bordeaux, France \\ b CNRS UMR5234, University of Bordeaux, Bordeaux,, France \\ c Emerging Antibiotic Resistance Unit, Medical and Molecular Microbiology, Department of Medicine, University of Fribourg, Fribourg, Switzerland \\ ' Swiss National Reference Center for Emerging Antibiotic Resistance, Fribourg, Switzerland \\ e INSERM European Unit (LEA), IAME, Paris, France \\ ${ }^{\mathrm{f}}$ University of Lausanne and University Hospital Center, Lausanne, Switzerland
}

Keywords:

MCR

Colistin

Resistance

Susceptibility testing

SuperPolymyxin medium

\begin{abstract}
The commercial SuperPolymyxin medium (ELITechGroup, Puteaux, France), currently licensed in Europe, is aimed to screen for either intrinsic or acquired colistin resistance in Gram negatives. It was tested for screening colistin-resistant Gram-negative rods from bacterial cultures of 145 colistin-resistant and 86 colistin-susceptible Gram-negative rods isolates. It exhibited a sensitivity and specificity of 95.2\% (138/145) and 95.3\% (82/86), respectively. The sensitivity for the detection of the 25 isolates carrying plasmid-mediated colistin resistance genes (mcr-1, mcr-2, mcr-3, and mcr-4) from bacterial cultures was of $100 \%$. It was also evaluated for the detection of colistin-resistant Gram-negative rods from 57 rectal swab samples (41 clinical rectal swab samples and from 16 spiked rectal swab samples supplemented with colistin-resistant enterobacterial isolates carrying $m c r$ genes). The overall sensitivity and specificity were of $100 \%$ (33/33) and $90.3 \%$ (56/62), respectively. The detection of isolates carrying plasmid-mediated $m c r$-like genes from rectal swabs was achieved with a lowest detection limit of $10^{3}$ to $10^{4} \mathrm{CFU} / \mathrm{mL}$. The SuperPolymyxin medium is adapted for screening and detection of colistin-resistant isolates from bacterial cultures and rectal swab samples, regardless of the level and the mechanism of colistin resistance. It might be useful in the context of prevention of outbreaks due to colistinresistant isolates and for epidemiological surveillance.
\end{abstract}

\section{Introduction}

The recently recognized global distribution of a self-transferable plasmid-borne colistin resistance determinant ( $m c r-1$ gene) (Liu et al., 2015; Poirel et al., 2017) raises a substantial public health concern because it fears a silent dissemination of this resistant trait. Horizontal transfers may therefore occur with this resistance trait by contrast to those resulting from chromosomal mutation. Once acquired by multidrug-resistant Gram-negative bacteria, it further limits treatment options for infected patients.

In this context, the European Centre for Disease Prevention and Control (ECDC) published on June 2016 released risk assessment caused by plasmid-mediated colistin resistance (www.ecdc.europa.eu). To reduce identified risks, it is proposed to improve laboratory detection of

\footnotetext{
* Corresponding author. Tel.: +41-26-300-9582; Fax: +33670023872.

E-mail address: laurent.poirel@unifr.ch (L. Poirel).

${ }^{1}$ Mailing address: Medical and Molecular Microbiology Unit, Department of Medicine, Faculty of Science, University of Fribourg, Chemin du Musée 18, CH-1700 Fribourg, Switzerland.
}

colistin resistance and in particular mcr genes detection, and to additionally initiate or further improve surveillance studies. ECDC recommends "to gather more information on the extent of the spread and the prevalence of gram-negative bacteria carrying the mcr-1 gene in human and animal microbiomes", and "to perform sentinel testing surveys". In health care facilities affected by sporadic cases or epidemic outbreaks of carbapenem-resistant and other multidrug-resistant Enterobacteriaceae, the recommendation is to perform "colistin susceptibility testing followed by PCR detection of the $m c r-1$ gene in colistinresistant isolates to ascertain the introduction of the $\mathrm{mcr}$-1 gene into their facility and ensure its containment by enhancing control measures such as contact isolation precautions, single-room isolation or cohorting".

In the meantime, several other plasmid-mediated colistin resistance genes ( $m c r-2$ (Xavier et al., 2016), $m c r-3$ (Yin et al., 2017), $m c r-4$ (Carattoli et al., 2017), and $m c r-5$ (Borowiak et al., 2017) have also been reported in Europe and China, suggesting that many plasmidmediated determinants might be responsible for colistin resistance. It is therefore problematic to rely only on molecular-based techniques for detection of plasmid-mediated colistin resistance, which detect only the previously identified genes. 
Moreover, susceptibility testing to colistin is currently challenging because the phenotypic methods usually used in routine laboratories for testing antimicrobials such as disk diffusion method and E-test system are not reliable for testing colistin susceptibility (Humphries, 2014). The broth microdilution reference method recommended by EUCAST (www.eucast.org) is often not implementable in routine practice because it requires manual preparation leading to risks of errors and qualified technicians, and is time consuming (Humphries, 2014).

Recently, the SuperPolymyxin screening medium was developed to detect acquired and intrinsic colistin-resistant Gram-negative rods from either bacterial colonies or clinical samples (Nordmann et al., 2016).

The objective of this study was to implement protocols for screening of colistin-resistant Gram-negative rods from bacterial colonies and from rectal swab samples using a commercial version of the SuperPolymyxin medium (ELITechGroup, Puteaux, France).

\section{Material and Methods}

\subsection{Colistin susceptibility testing with the BMD reference method}

The BMD method was performed according to the EUCAST guidelines (www.eucast.org). Briefly, BMD panels were prepared extemporaneously in 96-well sterile polystyrene microplates (Sarstedt, Nümbrecht, Germany). Dilutions of colistin (Sigma Aldrich, St Louis, MO, USA) ranging from 0.12 to $128 \mu \mathrm{g} / \mathrm{mL}$ were made in cationadjusted MH broth (Bio-Rad, Marnes-la-Coquette, France), without any addition of polysorbate 80 (Tween 80 ). A final concentration of $5.10^{5} \mathrm{CFU} / \mathrm{mL}$ of bacteria was added in each well, and the minimum inhibitory concentrations (MICs) were read after 16 to 20 hours of incubation at $35 \pm 2{ }^{\circ} \mathrm{C}$ in ambient air. Results were interpreted according to the EUCAST breakpoints; that is, isolates with MICs of colistin $\leq 2 \mu \mathrm{g} / \mathrm{mL}$ were categorized as susceptible, and those with MICs $>2 \mu \mathrm{g} / \mathrm{mL}$ were categorized as resistant. Colistin-susceptible ATCC 25922 Escherichia coli and ATCC 27853 Pseudomonas aeruginosa strains were included in all the experiments.

\subsection{Molecular genotyping for colistin resistance}

Colistin-resistant enterobacterial isolates were screened for plasmid-mediated colistin resistance $m c r-1, m c r-2, m c r-3, m c r-4$, and mcr-5 genes as described previously (Borowiak et al., 2017; Carattoli et al., 2017; Liu et al., 2015; Poirel et al., 2017; Xavier et al., 2016; Yin et al., 2017). Chromosomally encoded mutations in genes responsible for colistin resistance ( $p m r A, p m r B$, phoP, phoQ $m g r B$, and $\operatorname{crrB}$ genes) were also searched as described previously (Beceiro et al., 2011; Cheng et al., 2016; Jayol et al., 2015b; Lee and Ko, 2014; Poirel et al., 2014; Quesada et al., 2015; Sun et al., 2009).

\subsection{Detection of colistin resistance from bacterial colonies with the SuperPolymyxin medium}

Two hundred thirty-five bacterial isolates were tested, including 2 Gram-positive bacteria, 2 yeasts, 145 colistin-resistant, and 86 colistin-susceptible Gram-negative rods (Table S1). Among the 145 colistin-resistant isolates, 20 were of genus naturally resistant to colistin (Proteus, Providencia, Morganella, Serratia, and Hafnia), 21 were of genus inconstantly susceptible to colistin (Enterobacter, Stenotrophomonas, Achromobacter), and 104 presented acquired resistance to colistin (Escherichia, Klebsiella, Salmonella, Pseudomonas, Acinetobacter). Identification was performed using the Microflex bench-top MALDI-TOF mass spectrometer (Brücker, Champs-sur-Marne, France).

The commercial version of the homemade SuperPolymyxin selective medium was used for that study (ELITech MICROBIO, Signes, France). To avoid inoculum effect, bacterial colonies were not streak directly onto the medium. A bacterial suspension with an optical density of 0.5
McFarland was prepared into sterile $0.9 \% \mathrm{NaCl}$ and then 10 -fold diluted. Ten microliters of this dilution was then transferred with a $10-\mu \mathrm{L}$ calibrated loop and streaked onto the medium with a rake. The inoculated plates were visually inspected after 24 and 48 hours of incubation at $35 \pm 2{ }^{\circ} \mathrm{C}$ to evaluate bacterial growth. The isolates were interpreted as colistin resistant if they grew on the SuperPolymyxin medium, while they were interpreted as colistin susceptible if they did not grow on this medium.

The results obtained with the SuperPolymyxin medium after 48 hours of incubation were compared to those obtained with the reference BMD method. Discrepancies of the medium were determined to assess its performance to detect colistin resistance. In case of discrepancy, the isolates were retested twice ( 3 assays for each test), and results obtained were considered for performance calculations. Unsolved discrepancies were then maintained in the database for performance evaluation. Errors were ranked as follows: a very major error was defined when isolates did not grow on the SuperPolymyxin medium after 48 hours of incubation at $35 \pm 2{ }^{\circ} \mathrm{C}$ but exhibited MIC $>2 \mu \mathrm{g} / \mathrm{mL}$ by the BMD method (false-susceptible result), whereas a major error was defined when isolates grew on the SuperPolymyxin medium after 24 or 48 hours of incubation at $35 \pm 2{ }^{\circ} \mathrm{C}$ but exhibited MICs $\leq 2 \mu \mathrm{g} / \mathrm{mL}$ by the BMD method (false-resistant result). The number of resistant isolates and the number of susceptible isolates were used as denominators for very major error and major error calculations, respectively.

\subsection{Determination of the lowest limit of detection of the SuperPolymyxin medium}

Ten isolates were tested, including 5 colistin-resistant and 5 colistinsusceptible Gram-negative rods (Table 1). Using an inoculum with an optical density of 0.5 McFarland standard (inoculum of $\approx 10^{8} \mathrm{CFU} /$ $\mathrm{mL}$ ), serial 10 -fold dilutions of the isolates were made in sterile $0.9 \%$ $\mathrm{NaCl}$, and $100-\mu \mathrm{L}$ portions were plated onto the SuperPolymyxin medium. To quantify the viable bacteria in each dilution, Luria Bertani agar plates were inoculated concomitantly with $100 \mu \mathrm{L}$ of suspension and were incubated overnight at $35 \pm 2{ }^{\circ} \mathrm{C}$. The number of viable colonies was counted after 24 and 48 hours of culture at $35 \pm 2{ }^{\circ} \mathrm{C}$. The sensitivity and specificity cutoff values were set at $10^{3} \mathrm{CFU} / \mathrm{mL}$; that is, a limit value of $10^{3} \mathrm{CFU} / \mathrm{mL}$ and above was considered not efficiently detected (Nordmann et al., 2012).

\subsection{Detection of fecal carriage of colistin-resistant isolates}

Forty-one rectal swabs from 41 patients hospitalized in various clinical settings at the Bordeaux University Hospital, France, were tested (Table 2). Rectal swabs were inoculated into $1 \mathrm{~mL}$ of BD ESwab transport medium (Becton-Dickinson, Le-Pont-de-Claix, France). Ten microliters of the inoculated medium was then transferred with a $10-\mu \mathrm{L}$ calibrated loop onto the SuperPolymyxin plate and streak with a rake to avoid inoculum effect. To determine the performance of the SuperPolymyxin agar medium, an Eosine Methylene Blue (EMB) plate was streak in parallel with the same protocol. The plates were incubated for 48 hours at $35 \pm 2{ }^{\circ} \mathrm{C}$ and visually inspected at 24 and 48 hours to evaluate the bacterial growth. Each aspect of colony growing on the 2 plates (the SuperPolymyxin selective medium and the EMB nonselective medium) was identified using the Microflex bench-top MALDITOF mass spectrometer (Brücker). MICs of colistin of Gram-negative isolates growing on the plates were determined by broth microdilution reference method as described previously (Nordmann et al., 2016), and the results were interpreted according to EUCAST guidelines (www. eucast.org). Each aspect of enterobacterial isolates of genus usually susceptible to colistin (i.e., Escherichia, Citrobacter, Pseudomonas) that grew on the SuperPolymyxin medium was restreak onto another SuperPolymyxin plate for confirmation. For this purpose, the isolate was diluted into $0.9 \% \mathrm{NaCl}$ (to avoid inoculum effect) until obtaining a density of $0.5 \mathrm{McFarland}$, and $10 \mu \mathrm{L}$ of this dilution was then transferred 
Table 1

Lowest limit of detection of the SuperPolymyxin agar medium.

\begin{tabular}{|c|c|c|c|c|c|c|}
\hline Strains & Species & Origin & MIC of colistin $(\mu \mathrm{g} / \mathrm{mL})$ & Colistin profile $^{\mathrm{a}}$ & Genotype & Lowest detection limit $(\mathrm{CFU} / \mathrm{mL})^{\mathrm{b}}$ \\
\hline LECL & K. pneumoniae & France & 16 & $\mathrm{R}$ & mgrB gene truncated by IS903b-like & $10^{1}$ \\
\hline BOUJ & E. coli & France & 8 & $\mathrm{R}$ & Plasmid-mediated $\mathrm{mcr}-1$ gene & $10^{1}$ \\
\hline 136 & S. enterica & Spain & 4 & $\mathrm{R}$ & Plasmid-mediated $m c r-4$-like gene & $10^{1}$ \\
\hline 15,308 & P. aeruginosa & France & 64 & $\mathrm{R}$ & - & $10^{1}$ \\
\hline Aba6 & A. baumannii & Switzerland & $>128$ & $\mathrm{R}$ & PmrB G260D & $10^{1}$ \\
\hline Reference & K. pneumoniae & ATCC 53153 & 0.25 & $\mathrm{~S}$ & / & $>10^{7}$ \\
\hline Reference & E. coli & ATCC 25922 & 0.25 & S & / & $>10^{7}$ \\
\hline CATH & E. cloacae & France & 0.5 & $\mathrm{~S}$ & 1 & $10^{6}$ \\
\hline Reference & P. aeruginosa & ATCC 27853 & 1 & $\mathrm{~S}$ & 1 & $5.10^{5}$ \\
\hline Aba2 & A. baumannii & Switzerland & $\leq 0.12$ & $\mathrm{~S}$ & 1 & $10^{7}$ \\
\hline
\end{tabular}

- means "not determined"; / means "not applicable".

a $\mathrm{R}=$ resistant; $\mathrm{S}=$ susceptible.

b The growth of the isolates on the SuperPolymyxin medium was similar at 24 and 48 hours.

and streak onto the SuperPolymyxin medium with a rake. The plates were read after 48 hours of incubation at $35 \pm 2{ }^{\circ} \mathrm{C}$.

\subsection{Performance of the SuperPolymyxin medium to detect plasmid-} mediated colistin resistant isolates from spiked rectal swab samples

Sixteen spiked rectal swab samples were also tested, containing a series of enterobacterial isolates possessing plasmid-mediated colistin resistance determinants (13 $\mathrm{mcr}-1,1 \mathrm{mcr}-2,1 \mathrm{mcr}-3$-like, and $1 \mathrm{mcr}$ 4-like positive isolates) (Table 3 ). Using an inoculum with a density of 0.5 McFarland (inoculum of $\approx 10^{8} \mathrm{CFU} / \mathrm{mL}$ ), serial 10 -fold dilutions of the isolates were made in sterile $0.9 \% \mathrm{NaCl}$. Spiked rectal swab samples were made by adding $100 \mu \mathrm{L}$ of each strain dilution to $900 \mu \mathrm{L}$ of rectal swab transport medium that was obtained from a healthy volunteer. The plates were streak as described previously. To verify that rectal swab medium did not originally contain colistin-resistant isolates, $100 \mu \mathrm{L}$ of a nonspiked rectal swab medium was also streak onto the SuperPolymyxin medium (negative control). The plates were incubated at $35 \pm 2{ }^{\circ} \mathrm{C}$ and the lowest limits of detection were determined after 48 hours of growth.

\section{Results and Discussion}

3.1. Detection of colistin resistance from bacterial colonies with the SuperPolymyxin medium

The features of the 235 bacterial isolates included in this study to evaluate the performance of the SuperPolymyxin medium are summarized in Table S1. The 145 colistin-resistant isolates of Gram-negative rods used in this study presented various levels of resistance (MICs ranging from 4 to $>128 \mu \mathrm{g} / \mathrm{mL}$ by BMD reference method) (Table S1). The tested isolates exhibited various genotypes conferring colistin resistance, that is, related to various chromosomal mutations, and/ or acquisition of plasmid-mediated genes ( $m c r-1, m c r-2, m c r-3$, and $m c r-4$ genes) (Table S1). The discrepancies and the performance of the SuperPolymyxin medium to test colistin susceptibility are presented in Table 4.

\subsubsection{Gram-positive and yeast isolates}

The selectivity of the SuperPolymyxin medium was good since no Gram-positive bacteria and yeast grew on the medium.

\subsubsection{Gram-negative rods isolates with natural resistance to colistin}

Out of the 20 isolates exhibiting intrinsic resistance to colistin, 19 grew on the SuperPolymyxin medium (sensitivity of 95\%). A single Hafnia alvei isolate (MIC of colistin at $8 \mathrm{mg} / \mathrm{L}$ ) was not detected. We speculated that this false-negative result might be related to an heteroresistant phenotype of the strain, and determination of the MIC of colistin by using an E-test strip confirmed that hypothesis by showing 2 subpopulations, 1 resistant and 1 susceptible (Jayol et al., 2015a).

\subsubsection{Gram-negative isolates with inconstant susceptibility to colistin}

Three of the 11 colistin-resistant Enterobacter spp. isolates were not detected (sensitivity of 72.7\%). Failures to detect colistin-resistant Enterobacter cloacae isolates in that case might also be related to a heteroresistant phenotype. Indeed, intrinsic heteroresistance in some E. cloacae clusters has previously been described (Guerin et al., 2016). In that latter study, it was shown that heteroresistant isolates could be observed only on a plate seeded with a high inoculum, considering that the colistin-resistant subpopulation is lower than the colistinsusceptible subpopulation. Accordingly, among the $8 \mathrm{E}$. cloacae isolates that grew on the medium in our study, less than 10 colonies did grow on the plate for 3 isolates (Guerin et al., 2016). No false resistance was observed with $E$. cloacae isolates.

Out of the 9 colistin-resistant Stenotrophomonas maltophilia isolates tested, 8 were detected with the SuperPolymyxin medium (sensitivity of $89 \%$ ). Noteworthy, 6 isolates grew only after 48 hours of incubation, confirming that a delay of incubation $>24$ hours is required, in particular for that species. Out of the 12 colistin-susceptible $S$. maltophilia isolates tested, 11 did not grow on the SuperPolymyxin medium (specificity of 92\%). The single colistin-susceptible S. maltophilia isolate growing on the medium actually presented an MIC at $1 \mu \mathrm{g} / \mathrm{mL}$ which is just below the breakpoint.

Finally, only a single colistin-resistant $A$. xylosoxidans isolate was tested, and it was well detected with the SuperPolymyxin medium.

\subsubsection{Gram-negative isolates with acquired resistance to colistin}

Most carbapenemase-producing enterobacterial isolates belong to E. coli and Klebsiella pneumoniae species. Evaluating their susceptibility to colistin is therefore of utmost importance. Here we confirmed the excellent performance of the SuperPolymyxin medium for categorization of isolates belonging to these 2 species, with both sensitivity and specificity being at $100 \%$. Noteworthy, a single colistin-resistant $K$. pneumoniae isolate possessing mutations in the MgrB protein grew only after 48 hours of incubation. It is worth highlighting that the 22 isolates carrying plasmid-mediated colistin resistance genes grew within 24 hours.

Three Salmonella sp. isolates carrying plasmid-mediated colistin resistance ( $m c r-1$ or $m c r-4)$ grew on the medium, but a single colistinresistant Salmonella isolate whose mechanism of resistance remains unknown was falsely identified as susceptible.

All the colistin-resistant $P$. aeruginosa isolates grew on the SuperPolymyxin medium after 24 hours of incubation, except a single isolate that grew after 48 hours (sensitivity of $100 \%$ at 48 hours). Three of the 27 colistin-susceptible P. aeruginosa isolates grew on the SuperPolymyxin medium and were thus falsely detected as colistin resistant (specificity of 89\%). Those latter isolates exhibited MICs of 1 or $2 \mu \mathrm{g} / \mathrm{mL}$, just below the breakpoint for colistin $(4 \mu \mathrm{g} / \mathrm{mL})$. 
Table 2

Comparative growths on the nonselective Eosine Methylene Blue medium (EMB) and the selective SuperPolymyxin (SP) medium from clinical rectal swab samples.

\begin{tabular}{|c|c|c|c|c|c|}
\hline \multirow[t]{2}{*}{ Sample } & \multicolumn{2}{|c|}{ Bacterial species growing on the EMB medium at $48 \mathrm{~h}$} & \multirow[t]{2}{*}{ Colistin profile } & \multirow{2}{*}{$\begin{array}{l}\text { Growth on the SP } \\
\text { medium at } 48 \mathrm{~h}^{\mathrm{a}}\end{array}$} & \multirow{2}{*}{$\begin{array}{l}\text { Growth after restreaking } \\
\text { on the SP medium }\end{array}$} \\
\hline & Species & $\begin{array}{l}\text { MIC of colistin by } \\
\text { BMD }(\mu \mathrm{g} / \mathrm{mL})\end{array}$ & & & \\
\hline \multirow[t]{2}{*}{1} & E. coli & $\leq 0.12$ & $\mathrm{~S}$ & - & \\
\hline & E. faecalis & ND & NA & - & \\
\hline \multirow[t]{3}{*}{4} & E. coli & $\leq 0.12$ & $\mathrm{~S}$ & - & \\
\hline & K. pneumoniae & $\leq 0.12$ & $\mathrm{~S}$ & - & \\
\hline & S. aureus & ND & NA & - & \\
\hline \multirow[t]{4}{*}{9} & E. coli & $\leq 0.12$ & $\mathrm{~S}$ & - & \\
\hline & P. aeruginosa & 0.5 & $\mathrm{~S}$ & - & \\
\hline & E. faecalis & ND & NA & - & \\
\hline & E. faecium & ND & NA & - & \\
\hline 10 & E. coli & $\leq 0.12$ & $S$ & - & \\
\hline \multirow[t]{4}{*}{11} & E. coli & $\leq 0.12$ & $\mathrm{~S}$ & - & \\
\hline & P. aeruginosa & 0.5 & $\mathrm{~S}$ & - & \\
\hline & E. faecium & ND & NA & - & \\
\hline & E. hirae & ND & NA & - & \\
\hline 13 & Staphylococcus spp. & ND & NA & - & \\
\hline & E. faecalis & ND & NA & - & \\
\hline & Candida spp. & ND & NA & - & \\
\hline 16 & E. coli & $\leq 0.12$ & $\mathrm{~S}$ & - & \\
\hline & K. pneumoniae & $\leq 0.12$ & $\mathrm{~S}$ & - & \\
\hline 17 & E. coli & $\leq 0.12$ & $\mathrm{~S}$ & - & \\
\hline 19 & E. coli & $\leq 0.12$ & $\mathrm{~S}$ & - & \\
\hline 20 & Candida spp. & ND & NA & - & \\
\hline 21 & E. coli & 0.25 & $\mathrm{~S}$ & - & \\
\hline & K. pneumoniae & $\leq 0.12$ & $\mathrm{~S}$ & - & \\
\hline & E. faecalis & ND & NA & - & \\
\hline 22 & E. coli & 0.25 & $\mathrm{~S}$ & - & \\
\hline & E. faecalis & ND & NA & - & \\
\hline 23 & Candida spp. & ND & NA & - & \\
\hline 24 & P. mirabilis & $>128$ & $\mathbf{R}$ & $+(>100$ colonies $)$ & \\
\hline & K. oxytoca & $\leq 0.12$ & $\mathrm{~S}$ & - & \\
\hline & E. faecalis & ND & NA & - & \\
\hline 25 & P. aeruginosa & 1 & $\mathrm{~S}$ & - & \\
\hline & E. faecalis & ND & $\mathrm{S}$ & - & \\
\hline 26 & P. stuartii & $>128$ & $\mathbf{R}$ & $+(2$ colonies $)$ & \\
\hline & H. alvei & 8 & $\mathbf{R}$ & $+(1$ colony $)$ & \\
\hline & E. coli & $\leq 0.12$ & $\mathrm{~S}$ & - & \\
\hline & E. faecium & ND & NA & - & \\
\hline 27 & P. mirabilis & $>128$ & $\mathbf{R}$ & $+(>100$ colonies $)$ & \\
\hline & C. freundii & $\leq 0.12$ & $\mathrm{~S}$ & $+(2$ colonies $)$ & No \\
\hline & E. coli & $\leq 0.12$ & $\mathrm{~S}$ & - & \\
\hline 28 & P. mirabilis & $>128$ & $\mathbf{R}$ & $+(>100$ colonies $)$ & \\
\hline & S. maltophilia & 8 & $\mathbf{R}$ & $+(>100$ colonies $)$ & \\
\hline & E. faecium & ND & NA & - & \\
\hline 29 & P. vulgaris & $>128$ & $\mathbf{R}$ & $+(2$ colonies $)$ & \\
\hline & E. coli & $\leq 0.12$ & $\mathrm{~S}$ & $+(1$ colony $)$ & No \\
\hline & E. faecalis & ND & NA & - & \\
\hline 30 & E. coli & $\leq 0.12$ & $\mathrm{~S}$ & - & \\
\hline & K. pneumoniae & $\leq 0.12$ & $\mathrm{~S}$ & - & \\
\hline & E. cloacae & $\leq 0.12$ & $\mathrm{~S}$ & - & \\
\hline 31 & P. mirabilis & $>128$ & $\mathbf{R}$ & $+(>100$ colonies $)$ & \\
\hline & E. coli & $\leq 0.12$ & $\mathrm{~S}$ & - & \\
\hline & E. faecalis & ND & NA & - & \\
\hline 32 & E. coli & $\leq 0.12$ & $S$ & - & \\
\hline & P. aeruginosa & 2 & $\mathrm{~S}$ & $+(>100$ colonies $)$ & Yes ( 1 colony) \\
\hline & E. faecalis & ND & NA & - & \\
\hline 33 & E. coli & $\leq 0.12$ & $\mathrm{~S}$ & - & \\
\hline & E. faecium & ND & NA & - & \\
\hline 34 & E. coli & $\leq 0.12$ & $\mathrm{~S}$ & - & \\
\hline & E. faecalis & ND & NA & - & \\
\hline 35 & C. freundii & $\leq 0.12$ & $\mathrm{~S}$ & $+(6$ colonies $)$ & No \\
\hline & E. cloacae & $\leq 0.12$ & $\mathrm{~S}$ & - & \\
\hline 36 & C. freundii & 0.5 & $\mathrm{~S}$ & & \\
\hline & E. faecalis & ND & NA & - & \\
\hline 37 & E. coli & 0.25 & $S$ & & \\
\hline & K. pneumoniae & 1 & $\mathrm{~S}$ & & \\
\hline & P. aeruginosa & 1 & $\mathrm{~S}$ & & \\
\hline & Comamonas herstersii & 8 & $\mathbf{R}$ & $+(6$ colonies $)$ & \\
\hline & Acinetobacter junii & 8 & $\mathbf{R}$ & $+(2$ colonies $)$ & \\
\hline & E. faecalis & ND & NA & - & \\
\hline 39 & E. durans & ND & NA & - & \\
\hline 40 & P. mirabilis & $>128$ & $\mathbf{R}$ & $+(>100$ colonies $)$ & \\
\hline & E. coli & 0.5 & $\mathrm{~S}$ & & \\
\hline
\end{tabular}


Table 2 (continued)

\begin{tabular}{|c|c|c|c|c|c|}
\hline \multirow[t]{2}{*}{ Sample } & \multicolumn{2}{|c|}{ Bacterial species growing on the EMB medium at $48 \mathrm{~h}$} & \multirow[t]{2}{*}{ Colistin profile } & \multirow{2}{*}{$\begin{array}{l}\text { Growth on the SP } \\
\text { medium at } 48 \mathrm{~h}^{\mathrm{a}}\end{array}$} & \multirow{2}{*}{$\begin{array}{l}\text { Growth after restreaking } \\
\text { on the SP medium }\end{array}$} \\
\hline & Species & $\begin{array}{l}\text { MIC of colistin by } \\
\text { BMD }(\mu \mathrm{g} / \mathrm{mL})\end{array}$ & & & \\
\hline & K. pneumoniae & 0.25 & S & & \\
\hline & E. cloacae & 0.25 & S & - & \\
\hline & E. faecalis & ND & NA & - & \\
\hline & S. haemolyticus & ND & NA & - & \\
\hline \multirow[t]{3}{*}{41} & H. alvei & 8 & $\mathbf{R}$ & $+(5$ colonies $)$ & \\
\hline & E. coli & 0.25 & $\mathrm{~S}$ & - & \\
\hline & Enterobacter spp. & 1 & S & - & \\
\hline \multirow[t]{2}{*}{42} & C. freundii & 0.5 & S & - & \\
\hline & E. faecalis & ND & NA & - & \\
\hline \multirow[t]{4}{*}{43} & P. aeruginosa & 1 & S & & \\
\hline & A. junii & 4 & $\mathbf{R}$ & $+(20$ colonies $)$ & \\
\hline & E. faecalis & ND & NA & - & \\
\hline & Staphylococcus spp. & ND & NA & - & \\
\hline \multirow[t]{3}{*}{45} & E. coli & 0.5 & $\mathrm{~S}$ & - & \\
\hline & K. pneumoniae & 0.25 & S & - & \\
\hline & P. aeruginosa & 1 & S & $+(>100$ colonies $)$ & No \\
\hline 46 & P. putida & 0.5 & S & - & \\
\hline \multirow{4}{*}{47} & E. coli & 0.25 & $\mathrm{~S}$ & - & \\
\hline & K. pneumoniae & 0.5 & $\mathrm{~S}$ & - & \\
\hline & C. freundii & 0.25 & $\mathrm{~S}$ & - & \\
\hline & Acinetobacter spp. & 1 & S & - & \\
\hline 48 & E. coli & 0.25 & $\mathrm{~S}$ & - & \\
\hline \multirow[t]{6}{*}{49} & M. morganii & $>128$ & $\mathbf{R}$ & $+(>100$ colonies $)$ & \\
\hline & P. rettgeri & $>128$ & $\mathbf{R}$ & $+(>100$ colonies $)$ & \\
\hline & E. coli & 0.25 & $\mathrm{~S}$ & - & \\
\hline & K. pneumoniae & 0.5 & $\mathrm{~S}$ & - & \\
\hline & E. faecalis & ND & NA & - & \\
\hline & S. haemolyticus & ND & NA & - & \\
\hline \multirow[t]{3}{*}{50} & K. pneumoniae & 0.5 & S & - & \\
\hline & H. alvei & 8 & $\mathbf{R}$ & $+(4$ colonies $)$ & \\
\hline & E. casseliflavus & ND & NA & - & \\
\hline \multirow[t]{4}{*}{52} & P. mirabilis & $>128$ & $\mathbf{R}$ & $+(>100$ colonies $)$ & \\
\hline & E. coli & 0.25 & $\mathrm{~S}$ & - & \\
\hline & Comamonas herstersii & 0.25 & $\mathrm{~S}$ & - & \\
\hline & E. faecalis & ND & NA & - & \\
\hline \multirow[t]{2}{*}{53} & E. coli & 0.25 & S & - & \\
\hline & K. pneumoniae & 0.25 & S & - & \\
\hline \multirow[t]{6}{*}{54} & E. coli & 0.25 & S & $+(1$ colony $)$ & No \\
\hline & C. freundii & 0.5 & S & - & \\
\hline & K. pneumoniae & 0.5 & S & - & \\
\hline & E. cloacae & 0.5 & S & - & \\
\hline & P. aeruginosa & 1 & $S$ & - & \\
\hline & Rothia terrae & ND & NA & - & \\
\hline
\end{tabular}

$\mathrm{ND}=$ not determinate; $\mathrm{NA}=$ not applicable; $+=$ growth $;-=$ absence of growth

Colistin-resistant isolates (MIC $>2 \mathrm{mg} / \mathrm{L}$ ) are in bold font.

Discordant results compared to broth microdilution method are shaded in gray.

a Same results at 24 hours, except for the samples 41 and 50 for $\mathrm{H}$. alvei that did not grow on the SuperPolymyxin medium after 24 hours of incubation but grew at 48 hours.

Table 3

Lowest limits of detection of the SuperPolymyxin medium for isolates presenting plasmid-mediated colistin resistance in spiked rectal swab samples.

\begin{tabular}{|c|c|c|c|c|c|}
\hline Strains & Species & Origin & Genotype & MIC of colistin $(\mu \mathrm{g} / \mathrm{mL})$ & Lowest limit of detection $(\mathrm{CFU} / \mathrm{mL})^{\mathrm{a}}$ \\
\hline MALB & K. pneumoniae & France & Plasmid-mediated $m c r-1$ gene & 8 & $10^{2}$ \\
\hline BOUJ & E. coli & France & Plasmid-mediated $m c r-1$ gene & 8 & $10^{2}$ \\
\hline SOLAN & E. coli & France & Plasmid-mediated $m c r-1$ gene & 8 & $10^{2}$ \\
\hline Af23 & E. coli & South Africa & Plasmid-mediated $m c r-1$ gene & 8 & $10^{2}$ \\
\hline Af24 & E. coli & South Africa & Plasmid-mediated $m c r-1$ gene & 4 & $10^{2}$ \\
\hline Af31 & E. coli & South Africa & Plasmid-mediated $m c r-1$ gene & 8 & $10^{2}$ \\
\hline Af40 & E. coli & South Africa & Plasmid-mediated $m c r-1$ gene & 8 & $10^{2}$ \\
\hline Af45 & E. coli & South Africa & Plasmid-mediated $m c r-1$ gene & 8 & $10^{2}$ \\
\hline Af48 & E. coli & South Africa & Plasmid-mediated $m c r-1$ gene & 8 & $10^{2}$ \\
\hline Af49 & E. coli & South Africa & Plasmid-mediated $m c r-1$ gene & 16 & $10^{2}$ \\
\hline 41,331 & E. coli & France & Plasmid-mediated $m c r-1$ gene & 8 & $10^{2}$ \\
\hline BELG & E. coli & Belgium & Plasmid-mediated $m c r-2$ gene & 4 & $10^{3}$ \\
\hline I112 & E. coli & France & Plasmid-mediated $m c r$-3-like gene & 4 & $10^{3}$ \\
\hline 136 & S. enterica & Spain & Plasmid-mediated $m c r-4$-like gene & 4 & $10^{2}$ \\
\hline 237 & S. enterica & Spain & Plasmid-mediated $m c r-1$ gene & 16 & $10^{2}$ \\
\hline ANTU & S. enterica & France & Plasmid-mediated $m c r-1$ gene & 8 & $10^{2}$ \\
\hline
\end{tabular}

a $\mathrm{CFU} / \mathrm{mL}$ of rectal swab samples. 
Table 4

Discrepancies and performance of the SuperPolymyxin agar medium.

\begin{tabular}{|c|c|c|c|c|}
\hline & \multirow{2}{*}{$\frac{\text { Discrepancies }}{\text { VME rate }}$} & \multirow[b]{2}{*}{ ME rate } & \multicolumn{2}{|l|}{ Performance } \\
\hline & & & Sensitivity & Specificity \\
\hline \multicolumn{5}{|c|}{ Genus naturally resistant to colistin } \\
\hline Proteus & $0 \%(0 / 2)$ & NA & $100 \%(2 / 2)$ & NA \\
\hline Providencia & $0 \%(0 / 1)$ & NA & $100 \%(1 / 1)$ & NA \\
\hline Morganella & $0 \%(0 / 1)$ & NA & $100 \%(1 / 1)$ & NA \\
\hline Serratia & $0 \%(0 / 1)$ & NA & $100 \%(1 / 1)$ & NA \\
\hline Hafnia & $6.7 \%(1 / 15)$ & NA & $93.3 \%(14 / 15)$ & NA \\
\hline \multicolumn{5}{|c|}{ Genus inconstantly susceptible to colistin } \\
\hline Enterobacter & $27.3 \%(3 / 11)$ & $0 \%(0 / 9)$ & $72.7 \%(8 / 11)$ & $100 \%(9 / 9)$ \\
\hline Stenotrophomonas & $11.1 \%(1 / 9)$ & $8.3 \%(1 / 12)$ & $88.9 \%(8 / 9)$ & $91.7 \%(11 / 12)$ \\
\hline Achromobacter & $0 \%(0 / 1)$ & NA & $100 \%(1 / 1)$ & NA \\
\hline \multicolumn{5}{|c|}{ Genus usually susceptible to colistin } \\
\hline Escherichia & $0 \%(0 / 36)$ & $0 \%(0 / 13)$ & $100 \%(36 / 36)$ & $100 \%(13 / 13)$ \\
\hline Klebsiella & $0 \%(0 / 44)$ & $0 \%(0 / 12)$ & $100 \%(44 / 44)$ & $100 \%(12 / 12)$ \\
\hline Citrobacter & ND & $0 \%(0 / 2)$ & ND & $100 \%(2 / 2)$ \\
\hline Salmonella & $25.0 \%(1 / 4)$ & $0 \%(0 / 2)$ & $75.0 \%(3 / 4)$ & $100 \%(2 / 2)$ \\
\hline Pseudomonas & $0 \%(0 / 0)$ & $11.1 \%(3 / 27)$ & $100 \%(7 / 7)$ & $88.9 \%(24 / 27)$ \\
\hline Acinetobacter & $76.9 \%(1 / 13)$ & $0 \%(0 / 9)$ & $92.3 \%(12 / 13)$ & $100 \%(9 / 9)$ \\
\hline All & & & $95.2 \%(138 / 145)$ & $95.3 \%(82 / 86)$ \\
\hline
\end{tabular}

$\mathrm{NA}=$ not applicable; VME = very major error (false susceptibility); ME = major error (false resistance).

Out of 13 colistin-resistant Acinetobacter baumannii isolates tested, only a single isolate with an MIC at $128 \mu \mathrm{g} / \mathrm{mL}$ did not grow. All the colistin-susceptible isolates were well categorized and therefore corresponding to a sensitivity and specificity of $92.3 \%$ and $100 \%$, respectively.

\subsection{Detection limit for the SuperPolymyxin medium}

The colistin-resistant isolates tested from bacterial cultures grew on the SuperPolymyxin medium in 24 hours with a lowest limit of detection of $10^{1} \mathrm{CFU} / \mathrm{mL}$, being therefore below the cutoff value, whereas the limit of detection of the colistin-susceptible strains ranged from $5.10^{5}$ to $>10^{7} \mathrm{CFU} / \mathrm{mL}$, above the cutoff value (Table 1 ).

3.3. Detection of colistin-resistant isolates from rectal swabs with the SuperPolymyxin medium

To determine the performance of the SuperPolymyxin medium to detect colistin-resistant isolates directly from rectal swab, 41 clinical rectal swabs samples were tested. The results of growth on the EMB nonselective medium and on the SuperPolymyxin were compared to determine the sensitivity and the specificity of the SuperPolymyxin medium (Table 2). All the colistin-resistant isolates found on the EMB medium grew on the SuperPolymyxin medium giving a sensitivity of $100 \%$. Out of the 41 rectal swab samples, 17 colistin-resistant isolates were found on the SuperPolymyxin plate (Table 2). Thirteen of those isolates belonged to genus naturally resistant to colistin (Proteus spp., Providencia spp., Hafnia spp.), with MICs ranging from 8 to $>128 \mu \mathrm{g} / \mathrm{mL}$. Two H. alvei isolates were obtained only after 48 hours. Incubation of the SuperPolymyxin plates for 48 hours was therefore needed to avoid failure of detection of those isolates. Noteworthy, the rate of fecal carriage with intrinsic colistin-resistant isolates (29\%) was similar to the rate described in 2016 in the same hospital (Saly et al., 2017). Four isolates (1 S. maltophilia, 2 Acinetobacter junii, and 1 Comamonas herstersii) showing acquired resistance to colistin (MIC at 4 or $8 \mu \mathrm{g} / \mathrm{mL}$ ) were also recovered on the SuperPolymyxin medium (Table 2).

Sixty-two colistin-susceptible isolates were recovered from the EMB plates (Table 2). Those isolates presented MICs ranging from $<0.12$ to $2 \mu \mathrm{g} / \mathrm{mL}$. Out of those 62 isolates, 6 (2 Citrobacter freundii, 2 E. coli, and $2 P$. aeruginosa) grew on the SuperPolymyxin medium (false-positive results) giving a specificity of $90.3 \%$. All those latter isolates but one (a single $P$. aeruginosa isolate) had MICs lower than $0.25 \mu \mathrm{g} / \mathrm{mL}$.
Noteworthy, after replicating the corresponding colonies again on a SuperPolymyxin plate, none of them could grow after 48 hours of incubation at $35 \pm 2{ }^{\circ} \mathrm{C}$ (Table 2). Our previous study revealed that colistinsusceptible isolates could grow on the SuperPolymyxin medium when inoculating $\geq 10^{6} \mathrm{CFU} / \mathrm{mL}$ (Nordmann et al., 2016). It is therefore likely that the growth of those 5 colistin-susceptible isolates was related to an inoculum effect.

A single colistin-susceptible $P$. aeruginosa isolate grew on the SuperPolymyxin medium, even after a new streaking (Table 2). However, this isolate had an MIC of colistin at $2 \mu \mathrm{g} / \mathrm{mL}$ (just below the EUCAST breakpoint).

To determine the performance of the SuperPolymyxin medium to detect isolates showing a plasmid-encoded colistin resistance, we also spiked 16 rectal swabs with enterobacterial isolates carrying $\mathrm{mcr}-1$, $m c r-2, m c r-3-l i k e$, and $m c r-4-$ like genes (Table 3). All the isolates grew on the SuperPolymyxin medium within 24 hours with a lowest limit of detection of $10^{2}-10^{3} \mathrm{CFU} / \mathrm{mL}$, corresponding to a low level of fecal carriage. Although we tested here a limited number of isolates, the SuperPolymyxin medium appeared to be efficient to detect colistin-resistant and MCR-producing isolates.

\section{Conclusion}

The SuperPolymyxin medium exhibited a sensitivity and specificity of $95.2 \%$ and $95.3 \%$, respectively, for detection of colistin-resistant isolates from bacterial culture. It exhibited an overall sensitivity and specificity of $100 \%$ and $90.3 \%$, respectively, for detection of colistinresistant isolates in rectal swab samples. Isolates carrying plasmid mediated colistin resistance ( $m c r-1$ to $m c r-4)$ grew after 24 hours of incubation and were thus detected with a sensitivity of $100 \%$ from both bacterial culture and rectal swab samples. Incubation of 48 hours was necessary to detect a few colistin-resistant isolates. The SuperPolymyxin medium therefore provides a useful tool for screening occurrence of colistin-resistant isolates from bacterial cultures but also from gut carriage, regardless of the level and mechanism of colistin resistance. Using such a selective medium might contribute to improve detection of colistin resistant isolates and consequently prevent their dissemination.

Supplementary data to this article can be found online at https://doi. org/10.1016/j.diagmicrobio.2018.05.008. 


\section{Funding}

This work was financed by grants of the University of Bordeaux (France), the University of Fribourg (Switzerland), and by the Swiss National Reference Center for Emerging Antibiotic Resistance (NARA).

\section{References}

Beceiro A, Llobet E, Aranda J, Bengoechea JA, Doumith M, Hornsey M, et al. Phosphoethanolamine modification of lipid A in colistin-resistant variants of Acinetobacter baumannii mediated by the PmrAB two-component regulatory system. Antimicrob Agents Chemother 2011;55:3370-9. (doi:AAC.00079-11 [pii]) https:// doi.org/10.1128/AAC.00079-11.

Borowiak M, Fischer J, Hammerl JA, Hendriksen RS, Szabo I, Malorny B. Identification of a novel transposon-associated phosphoethanolamine transferase gene, $\mathrm{mcr}-5$, conferring colistin resistance in d-tartrate fermenting Salmonella enterica subsp. enterica serovar Paratyphi B. J Antimicrob Chemother 2017;72:3317-24. https://doi.org/10. 1093/jac/dkx327.

Carattoli A, Villa L, Feudi C, Curcio L, Orsini S, Luppi A, et al. Novel plasmid-mediated colistin resistance mcr-4 gene in Salmonella and Escherichia coli, Italy 2013, Spain and Belgium, 2015 to 2016. Euro Surveill 2017;22. https://doi.org/10.2807/1560-7917. ES.2017.22.31.30589

Cheng YH, Lin TL, Lin YT, Wang JT. Amino acid substitutions of CrrB responsible for resistance to colistin through $\mathrm{CrrC}$ in Klebsiella pneumoniae. Antimicrob Agents Chemother 2016;60:3709-16. https://doi.org/10.1128/AAC.00009-16.

Guerin F, Isnard C, Sinel C, Morand P, Dhalluin A, Cattoir V, et al. Cluster-dependent colistin hetero-resistance in Enterobacter cloacae complex. J Antimicrob Chemother 2016; 71:3058-61. (doi:dkw260 [pii]) https://doi.org/10.1093/jac/dkw260.

Humphries RM. Susceptibility testing of the polymyxins: where are we now? Pharmacotherapy 2014. https://doi.org/10.1002/phar.1505.

Jayol A, Nordmann P, Brink A, Poirel L. Heteroresistance to colistin in Klebsiella pneumoniae associated with alterations in the PhoPQ regulatory system. Antimicrob Agents Chemother 2015a;59:2780-4. (doi:AAC.05055-14 [pii]) https://doi.org/10. 1128/AAC.05055-14.

Jayol A, Poirel L, Villegas MV, Nordmann P. Modulation of $\operatorname{mgrB}$ gene expression as a source of colistin resistance in Klebsiella oxytoca. Int J Antimicrob Agents 2015b;46: 108-10. (doi:S0924-8579(15)00109-0 [pii]) https://doi.org/10.1016/j.jjantimicag. 2015.02.015.
Lee JY, Ko KS. Mutations and expression of PmrAB and PhoPQ related with colistin resistance in Pseudomonas aeruginosa clinical isolates. Diagn Microbiol Infect Dis 2014;78 271-6. (doi:S0732-8893(13)00640-8 [pii]) https://doi.org/10.1016/j.diagmicrobio. 2013.11.027.

Liu YY, Wang Y, Walsh TR, Yi LX, Zhang R, Spencer J, et al. Emergence of plasmidmediated colistin resistance mechanism MCR-1 in animals and human beings in China: a microbiological and molecular biological study. Lancet Infect Dis 2015;16: 161-8. (doi:S1473-3099(15)00424-7 [pii]) https://doi.org/10.1016/S1473-3099 (15)00424-7.

Nordmann P, Girlich D, Poirel L. Detection of carbapenemase producers in Enterobacteriaceae by use of a novel screening medium. J Clin Microbiol 2012;50:2761-6. (doi: JCM.06477-11 [pii]) https://doi.org/10.1128/JCM.06477-11.

Nordmann P, Jayol A, Poirel LA. Universal culture medium for screening polymyxinresistant gram negatives isolates. J Clin Microbiol 2016;54:1395-9. https://doi.org/ 10.1128/JCM.00446-16.

Poirel L, Jayol A, Bontron S, Villegas MV, Ozdamar M, Turkoglu S, et al. The mgrB gene as a key target for acquired resistance to colistin in Klebsiella pneumoniae. Antimicrob Chemother 2014;70:75-80. (doi:dku323 [pii]) https://doi.org/10. 1093/jac/dku323.

Poirel L, Jayol A, Polymyxins Nordmann P. Antibacterial activity, susceptibility testing, and resistance mechanisms encoded by plasmids or chromosomes. Clin Microbiol Rev 2017;30:557-96. https://doi.org/10.1128/CMR.00064-16.

Quesada A, Porrero MC, Tellez S, Palomo G, Garcia M, Dominguez L. Polymorphism of genes encoding PmrAB in colistin-resistant strains of Escherichia coli and Salmonella enterica isolated from poultry and swine. J Antimicrob Chemother 2015;70:71-4. (doi:dku320 [pii]) https://doi.org/10.1093/jac/dku320.

Saly M, Jayol A, Poirel L, Megraud F, Nordmann P, Dubois V. Prevalence of faecal carriage of colistin-resistant Gram-negative rods in a university hospital in western France, 2016. J Med Microbiol 2017;66:842-3. https://doi.org/10.1099/jmm.0. 000497.

Sun S, Negrea A, Rhen M, Andersson DI. Genetic analysis of colistin resistance in Salmonella enterica serovar Typhimurium. Antimicrob Agents Chemother 2009;53. 2298-305. doi:AAC.01016-08 [pii] https://doi.org/10.1128/AAC.01016-08.

Xavier BB, Lammens C, Ruhal R, Kumar-Singh S, Butaye P, Goossens H, et al. Identification of a novel plasmid-mediated colistin-resistance gene, mcr-2, in Escherichia coli, Belgium, June 2016. Euro Surveill 2016;21. https://doi.org/10.2807/1560-7917.ES. 2016.21.27.30280. (30280 [pii]).

Yin W, Li H, Shen Y, Liu Z, Wang S, Shen Z, et al. Novel plasmid-mediated colistin resistance gene mcr-3 in Escherichia coli. MBio 2017;8. https://doi.org/10.1128/mBio. 00543-17. 